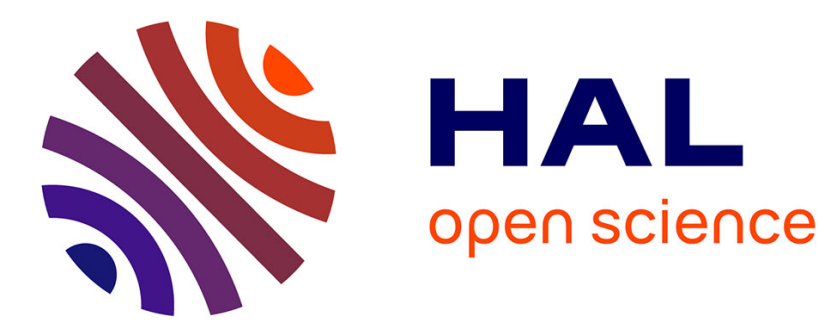

\title{
On the use of inner position loop in co-manipulation task
} Sylvain Devie, Pierre-Philippe Robet, Yannick Aoustin, Maxime Gautier

\section{To cite this version:}

Sylvain Devie, Pierre-Philippe Robet, Yannick Aoustin, Maxime Gautier. On the use of inner position loop in co-manipulation task. ROMANSY 22-Robot Design, Dynamics and Control Proceedings of the 22nd CISM IFToMM Symposium, June 25-28, 2018, Rennes, France, 2019. hal-01984436

\section{HAL Id: hal-01984436 https://hal.science/hal-01984436}

Submitted on 17 Aug 2019

HAL is a multi-disciplinary open access archive for the deposit and dissemination of scientific research documents, whether they are published or not. The documents may come from teaching and research institutions in France or abroad, or from public or private research centers.
L'archive ouverte pluridisciplinaire HAL, est destinée au dépôt et à la diffusion de documents scientifiques de niveau recherche, publiés ou non, émanant des établissements d'enseignement et de recherche français ou étrangers, des laboratoires publics ou privés. 


\title{
On the use of inner position loop in co-manipulation task
}

\author{
Sylvain Devie, Pierre-Philippe Robet, Yannick Aoustin, and Maxime Gautier \\ Université de Nantes Laboratoire des Sciences du Numérique de Nantes, \\ UMR 6004, CNRS, \\ sylvain.devie@univ-nantes.fr
}

\begin{abstract}
This paper deals with the interaction between a specified robot and its environment. A particular case is considered, which is a comanipulation case, where an operator is performing a co-operative task with the robot. Cascaded loops are considered for the control design and a frequency analysis is performed to study the influence of the position loop.
\end{abstract}

Keywords: Force control, Co-manipulation, Control design

\section{Introduction}

The capability of robot systems to perform advanced tasks in unstructured and imprecise environments is strongly dependent on their ability to simultaneously control end-effector motions and active forces. Decades ago, the improvement of formal algorithms that efficiently computed robot models [3] allowed for a good identification of robots to efficiently design and calculate their controllers. This paper focuses on the design of a control law for both force control and co-manipulation tasks.

An efficient control law mixing position and force control is presented in [5] by Khatib et al. and the frequency limits of these kinds of control laws are presented by Khatib in [4]. Two different configurations have been proposed for the closed loops according to the position of the force loop: this loop can be the inner or the outer loop. In [6], an external force loop is proposed in order to encapsulate an inner impedance loop. It also proposed a compensation of the environmental displacement, which is one of the main problems of the conventional external loop. In [7], they proposed a control law using an inner force loop encapsulated into an outer impedance loop carried out by vision. This solution presents an independence between both loops, which allows the user to tune them separately and choose the best design for each of them.

Here, the configuration of the robot leads us to consider an outer force loop which encapsulates an inner velocity loop as presented in [2]. The main advantages of this control law are its simplicity and the fact it can be easily tuned into an equivalent impedance control law [1]. However, this configuration limits the control of the position and does not permit the utilization of virtual guide. The 
solution proposed in this paper is to add an intermediate position loop between the inner velocity loop and the outer force loop. But if this extra loop can improve the position control, it also affects the bandwidth of the system. The aim of this paper is to study the influence of this intermediate loop and how it can affect the co-manipulation task.

This paper is outlined as follows. Section 2 defines the mechanical part and the controller design of the studied system. Sections 3 presents theoretical study. Section 4 details our experiments. Section 5 offers our conclusions.

\section{System definition}

\subsection{Mechanical part}

Let us consider a simple one degree of freedom prismatic robot, interacting with the environment thanks to a force sensor. In this study, the considered force sensor is composed of a spring with a stiffness coefficient $K_{r 12}$ checked experimentally. The considered system is represented in Fig. 1. It is a two degrees of freedom robot. The first degree of freedom $q_{1}$ is the position of the end effector of the robot, this position can be measured and controlled. The second degree of freedom $x$ is the position of the extremity of the force sensor interacting with the environment. The relative deformation of the spring is defined thanks to these positions by: $q_{2}=x-q_{1}$. All the positions are defined in meters $(m)$. This force sensor measures the force $\tau_{e x t}=K_{r 12} q_{2}$.

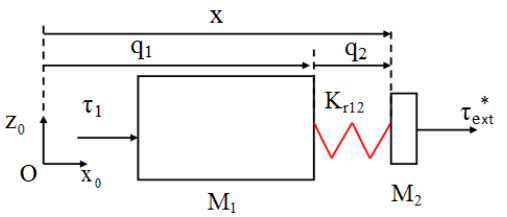

(a) Parameters of the EMPS

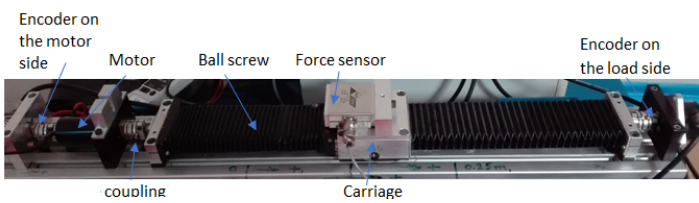

(b) Main components of the EMPS

Fig. 1: Definition of the considered system

Body 1 represents the moving part of the robot, including the rotor of the motor, the power transmission gear and an attached end of the force sensor. Body 2 represents the other extremity of the force sensor and the tool, which is in contact with the environment. For each body $i=[1,2], M_{i}$ is the mass in translation $(k g), F_{v i}$ is the viscous friction coefficient $(\mathrm{N} /(\mathrm{m} / \mathrm{s}))$ and $F_{c i}$ is the Coulomb friction $(N)$. For position $q_{i}$, the velocities $(\mathrm{m} / \mathrm{s})$ and the accelerations $\left(\mathrm{m} / \mathrm{s}^{2}\right)$ are respectively called $\dot{q}_{i}$ and $\ddot{q}_{i}$.

In order to avoid resonance issues, the frequencies of the dynamic system will be chosen to be smaller than $10 \%$ of the first flexible mode of the sensor: 
$\sqrt{K_{r 12} / M_{2}}$. With respect to the reference frame fixed to the robot, the dynamic model of the mechanical device is as follows

$$
\begin{aligned}
& \tau_{1}=M_{1} \ddot{q}_{1}+F_{v 1} \dot{q}_{1}+F_{c 1} \operatorname{sign}\left(\dot{q}_{1}\right)+\tau_{\text {ext }} \\
& 0=M_{2} \ddot{x}-\tau_{\text {ext }}+F_{v 2} \dot{x}+F_{c 2} \operatorname{sign}(\dot{x})+\tau_{\text {ext }}^{*}
\end{aligned}
$$

with $\tau_{1}$ the actuation force on body 1 and $\tau_{e x t}^{*}$ the interaction force from the environment on body 2 .

The force $\tau_{\text {ext }}(N)$ measured by the sensor and the velocity $\dot{x}$ are linked by the impedance of the environment: $Z_{e}=\tau_{e x t}^{*} / \dot{x}$. If the robot is controlled in order to apply a specific force on the environment, $Z_{e}(\mathrm{~N} /(\mathrm{m} / \mathrm{s}))$ is the impedance of this environment. Three particular cases can be considered for the impedance. The softest case is the free case: $\tau_{e x t}^{*}=0$ so $Z_{e}=0$, which means that there is no obstacle and the robot is free to move. The hardest case is the constraint case: $\dot{x}=0$ so $Z_{e} \longrightarrow \infty$, which means that the environment is an infinitely rigid obstacle. The last case is an intermediate case, where $0<Z_{e}$.

\subsection{Control design}

In a previous study [2], a simple control law was proposed to do co-manipulation task on the system presented in Fig. 1. This specific application needs a system fast enough to be as transparent as possible for the operator. That means the operator should be able to move the robot without been limited by its dynamic or its response time.

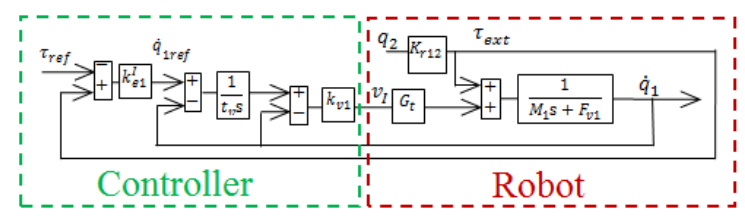

(a) Case without position loop.

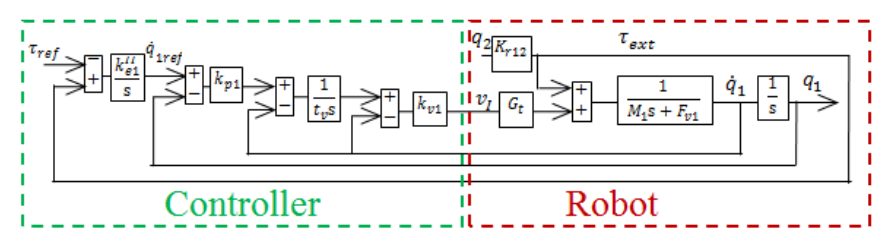

(b) Case with position loop.

Fig. 2: Cascaded closed loop of speed, position and force in the case of comanipulation.

The environmental impedance depends on the impedance of the environment and on the impedance of the operator's hands and is supposed to be unknown. 
In this case, the control system is illustrated in Fig. 2a. The frictions $F_{c 2} \operatorname{sign}(\dot{x})$ and $F_{v 2} \dot{x}$ are neglected with respect to $\tau_{e x t}$. In this case, the following equations describe the dynamics of the closed loop system:

$$
\begin{aligned}
& \tau_{1}=M_{1} \ddot{q}_{1}+F_{v 1} \dot{q}_{1}+F_{c 1} \operatorname{sign}\left(\dot{q}_{1}\right)+\tau_{e x t} \\
& 0=M_{2} \ddot{x}-\tau_{\text {ext }}+\tau_{\text {ext }}^{*}
\end{aligned}
$$

In the following, we consider $M_{2} \ddot{x} \ll \tau_{e x t}^{*}$. In this case we have $\tau_{\text {ext }}^{*}=\tau_{\text {ext }}$.

In the following, all the calculations will be done based on the control laws presented in Figs. 2a and 2b. It is important to specify that, for the rest of this paper, the Coulomb friction $F_{c 1}$ is considered as a constant perturbation corrected by the integral correction, and it is not taken into account in the calculation. Two cases are considered for the control law:

- A case without position loop, with IP correction for the velocity loop and P correction for the force loop. This case is presented in Fig. 2a.

- A case with a position loop, with still IP correction for the velocity loop. An intermediate position loop is added with a $\mathrm{P}$ correction. The outer force loop has an I correction. This case is presented in Fig. 2b.

In these control laws, the velocity and the position loop are used to control the performance of the system and the force loop is mostly used to control its transparency. Here, by increasing the gain $k_{e 1}^{I}$ or $k_{e 1}^{I I}$ we increase the transparency. However, a very high gain can lead to the instability. These two control structures will be studied in the next section.

In the following, the subscript $v, p$ and $e$ will respectively identify the variables related to the velocity, the position and the effort. The superscript ${ }^{I}$ is used for the cases using no position loop while the superscript ${ }^{I I}$ is for the case using the position loop.

\section{Calculation}

Let us now consider the co-manipulation case. In this case, the environmental impedance is unknown. We choose to use the same gain $k_{e 1}^{I}$ as defined in [2].

In the cases of a classical rigid industrial robot, the high reduction ratio of the transmission gear and the large inertia gives us $\tau_{1}>>\tau_{\text {ext }}$. Also, we ensure that the velocity loop is well calculated and has a frequency sufficiently higher than the external perturbation to do two hypothesis. The first hypothesis is a linear relation between the external forces and the velocity : $\tau_{e}=1 / k_{e 1} \dot{q}_{1}=k_{c} \dot{q}_{1}$. The second hypothesis is that the external force can be neglected in the calculation of the inner velocity loop coefficients.

The goal of this section is to calculate the correction gains and the bandwidths of the co-manipulation control with and without the position loop. For the calculation of the correction gains, the bandwidth of the velocity loop is imposed to $\omega_{v}(\mathrm{rad} / \mathrm{s})$ and the phase margins $\phi(\mathrm{rad})$ are imposed for all the loops. 


\subsection{Inner velocity loop}

The inner velocity loop is common to the two control laws. According to Figs. $2 \mathrm{a}$ and $2 \mathrm{~b}$, the open loop transfer function is given by the equation:

$$
T_{v o}(\mathrm{~s})=\frac{\dot{q}_{1}}{\dot{q}_{1 r e f}-\dot{q}_{1}}=\frac{k_{v 1} G_{\tau}}{t_{v} \mathrm{~s}} \frac{1}{M_{1} \mathrm{~s}+F_{v 1}+k_{v 1} G_{\tau}}
$$

When there is no position loop, it is defined by $k_{c}^{I}=1 / k_{e 1}^{I}$. In the other case, it is defined by $k_{c}^{I I}=1 / k_{e 1}^{I I}$

Imposing $T_{v o}\left(j \omega_{0 v}\right)=1 \mathrm{e}^{j\left(-\pi+\phi_{v}\right)}$ leads to the following values of $k_{v 1}$ and $t_{v}$ :

$$
k_{v 1}=\frac{M_{1} \omega_{0 v} \tan \left(\phi_{v}\right)-F_{v 1}}{G_{\tau}} ; t_{v}=\frac{k_{v 1} G_{\tau}}{M_{1} \omega_{0 v}^{2}} \cos \left(\phi_{v}\right)
$$

Because of the common part of the two control laws, the correction gains of the velocity loop will be the same both for the case with the position loop and for the case without the position loop.

The closed loop transfer function is the following for the velocity:

$$
T_{v c}(\mathrm{~s})=\frac{\dot{q}_{1}}{\dot{q}_{1 r e f}}=\frac{1}{1+\left(t_{v}+\frac{F_{v 1}}{K_{v}}\right) \mathrm{s}+\frac{M_{1}}{K_{v}} \mathrm{~s}^{2}}=\frac{1}{1+\frac{2 z_{v}}{\omega_{0 v}} \mathrm{~s}+\frac{\mathrm{s}^{2}}{\omega_{0 v}^{2}}}
$$

with $K_{v}=k_{v 1} G_{\tau} / t_{v}, \omega_{0 v}=\sqrt{K_{v} / M_{1}}$ and $z_{v}=\left(t_{v}+\frac{F_{v 1}}{K_{v}}\right) \frac{\omega_{0 v}}{2}$.

\subsection{Outer force loop}

In the case with no position loop, the outer force loop has the following open loop equation:

$$
T_{e o}^{I}(\mathrm{~s})=\frac{\tau_{e x t}}{\tau_{r e f}-\tau_{e x t}}=\frac{k_{e 1}^{I} k_{c}^{I}}{1+\frac{2 z_{v}}{\omega_{0 v}} \mathrm{~s}+\frac{\mathrm{s}^{2}}{\omega_{0 v}^{2}}}=\frac{K_{e_{c}}^{I}}{1+\frac{2 z_{v}}{\omega_{0 v}} \mathrm{~s}+\frac{\mathrm{s}^{2}}{\omega_{0 v}^{2}}}
$$

with $K_{e_{c}}^{I}=k_{e 1}^{I} k_{c}^{I}$

According to [2] $k_{c}^{I}$ is defined with $k_{c}^{I}=1 / k_{e 1}^{I}$, which means $K_{e_{c}}^{I}=1$, $z_{e_{c}}^{I}=z_{v} / \sqrt{2}$ and $\omega_{0 e_{c}}^{I}=\omega_{0 v} \sqrt{2}$. According to this relation, the cut-off frequency of the force loop is supposed to be greater than the one of the velocity loop. However, according to our model, the bandwidth of the force loop is supposed to be far smaller than the cut-off frequency of the velocity loop. This result shows the limits of this model: it can be used to easily calculate the correction gains of the system. However, the bandwidth of the force loop has to be calculated experimentally. 


\subsection{Intermediate position loop and outer force loop}

Similar to the previous section, when a position loop is added between the velocity loop and the force loop, the transfer function of this new loop is:

$$
T_{p o}^{I I}(\mathrm{~s})=\frac{q_{1}}{q_{r e f}-q_{1}}=\frac{k_{p 1} / \mathrm{s}}{1+\frac{2 z_{v}}{\omega_{0 v}} \mathrm{~s}+\frac{\mathrm{s}^{2}}{\omega_{0 v}^{2}}}
$$

Imposing $T_{p o}\left(j \omega_{p}\right)=1 \mathrm{e}^{j\left(-\pi+\phi_{p}\right)}$ leads us to the following cut-off frequency $\omega_{0 p}$ and correction gain $k_{p 1}$ :

$\omega_{0 p}=\omega_{0 v}\left(-z_{v} \tan \left(\phi_{p}\right)+\sqrt{1+\tan ^{2}\left(\phi_{p}\right) z_{v}^{2}}\right) ; k_{p 1}=\omega_{0 p} \sqrt{\left(1-\left(\frac{\omega_{0 p}}{\omega_{0 v}}\right)^{2}\right)^{2}+\left(2 z_{v} \frac{\omega_{0 p}}{\omega_{0 v}}\right)^{2}}$

The equation of the closed position loop is the following:

$$
T_{p c}^{I I}(\mathrm{~s})=\frac{q_{1}}{q_{r e f}}=\frac{1}{1+\frac{\mathrm{s}}{k_{p 1}}+2 \frac{z_{v}}{\omega_{0 v} k_{p 1}} \mathrm{~s}^{2}+\frac{\mathrm{s}^{3}}{\omega_{0 v}^{2} k_{p 1}}}=\frac{1}{\left(1+\tau_{p} \mathrm{~s}\right)\left(1+\frac{2 z_{p}}{\omega_{0 p}} \mathrm{~s}+\frac{\mathrm{s}^{2}}{\omega_{0 p}^{2}}\right)}
$$

In this case, we have $1 / \tau_{p}>>\omega_{0 p}$, so:

$$
T_{p c}^{I I}(\mathrm{~s})=\frac{1}{1+\frac{2 z_{p}}{\omega_{0 p}} \mathrm{~s}+\frac{\mathrm{s}^{2}}{\omega_{0 p}^{2}}}
$$

The transfer function of the open force loop is the following:

$$
T_{e o}^{I I}(\mathrm{~s})=\frac{\tau_{e x t}}{\tau_{r e f}-\tau_{e x t}}=k_{c}^{I I} k_{e 1}^{I I} T_{p c}^{I I}(\mathrm{~s})=\frac{k_{c}^{I I} k_{e 1}^{I I}}{1+\frac{\mathrm{s}}{k_{p 1}}+2 \frac{z_{v}}{\omega_{0 v} k_{p 1}} \mathrm{~s}^{2}+\frac{\mathrm{s}^{3}}{\omega_{0 v}^{2} k_{p 1}}}
$$

However, using the simplified form (10), this equation can be written in the following form:

$$
T_{e o}^{I I}(\mathrm{~s})=\frac{K_{e_{c}}^{I I}}{1+\frac{2 z_{p}}{\omega_{0 p}} \mathrm{~s}+\frac{\mathrm{s}^{2}}{\omega_{0 p}^{2}}}
$$

with $K_{e_{c}}^{I I}=k_{c}^{I I} k_{e 1}^{I I}$

\section{Experiments and discussion}

\subsection{Numerical application}

The EMPS robot is used. For $\omega_{0 v}=150 \mathrm{rad} / \mathrm{s}, \phi_{v}=83 \mathrm{deg}, \phi_{p}=82 \mathrm{deg}$ and $\phi_{e}=85 \mathrm{deg}$, we get the following coefficients for the correction: 
$k_{v 1}=978 \mathrm{~V} /(\mathrm{m} / \mathrm{s}), t_{v}=6.010^{-3} \mathrm{~s}$ and $k_{e 1}^{I}=2.310^{-3}(\mathrm{~m} / \mathrm{s}) / \mathrm{N}$ for the case without position loop, $k_{p 1}=43 \mathrm{~s}^{-1}$ and $k_{e 1}^{I I}=6.110^{-4}(\mathrm{~m} / \mathrm{s}) / N$ for the case with position loop.

These results are illustrated by the Nichols diagram of the open loop transfer function for the force loop. Because of the hypothesis of this model, this diagram matches with reality for low frequencies. In this case, it is enough to ensure the stability of the system and a good performance in this area of frequencies. However, it does not allow us to calculate the bandwidth of the real system, but only theoretical values for the model.

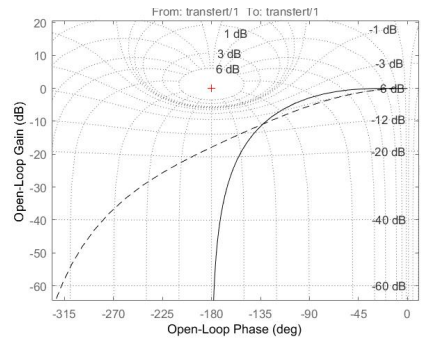

(a) Nichols diagram for the open force loop, from the numerical application

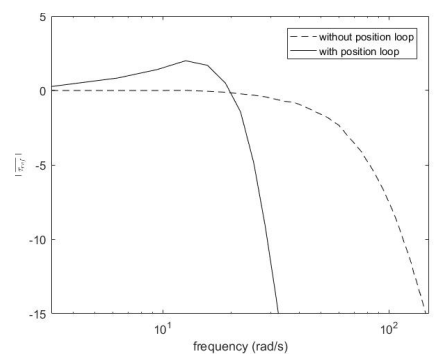

(b) Amplitude of the applied force for a sinusoidal input function of the frequency $\omega$, from the experimental validation

Fig. 3: Behaviour without position loop (full) and with position loop (dashed)

\subsection{Experimental validation}

Experimental validations were performed on the EMPS robot. During these experiments, the worst case for the environmental impedance is considered to tune $k_{e 1}$. The extremity of the force sensor was fixed to the base of the robot and a specific input $\tau_{r e f}$ was applied. Because a human operator cannot apply a precise signal, it has been digitally simulated with an input signal. This input is a sinusoidal signal with an amplitude equal to 1 and a frequency between 1 and $150 \mathrm{rad} / \mathrm{s}$. In this case, the bandwidth of the force loop is equal to $65 \mathrm{rad} / \mathrm{s}$ for the case without force loop and $23 \mathrm{rad} / \mathrm{s}$ for the case with force loop. These results are presented in Fig. 3b.

While the intermediate position loop allows us to perform a virtual guide, it decreases the bandwidth of the force loop and limits the applications of the system. The response time of the human hand is up to $310 \mathrm{~ms}$ for a visual stimulus. This estimation gives us a bandwidth at around $20 \mathrm{rad} / \mathrm{s}$. However, in the considered case, the bandwidth of the force loop considering an inner position loop 
$(23 \mathrm{rad} / \mathrm{s})$ is higher than this value. That means the co-manipulation operation is still possible in this configuration.

Another classical approach for co-manipulation performances is the impedance control law. In [1], we have shown an equivalence between this control law and the one presented in this paper without inner force loop. thanks to this equivalence, both controllers can be tuned to have the same performances, and almost the same bandwidth $(70 \mathrm{rad} / \mathrm{s})$.

\section{Conclusion}

In this paper, a simple control law was proposed to perform a co-manipulation task. Two different cases were considered, depending on the use of a position loop. The aim of adding this loop was to be able to precisely control the trajectory of the robot's end-effector. A frequency analysis was performed and has shown that the use of the position loop reduces the bandwidth of the force loop. This reduction limits the applications of the control law but still allows for low-frequency applications.

\section{Acknowledgments}

This research was supported by BPI France through the Competitiveness Clusters Structuring Project (PSPC) MammoNExT. The project is co-funded by the European Union. Europe is now investing in Pays de la Loire with the European Regional Development Fund.

\section{References}

1. Devie, S., Robet, P.p., Aoustin, Y., Gautier, M.: Impedance control using a cascaded loop force control. IEEE Robotics \& Automation letter pp. 1-7 (2018)

2. Devie, S., Robet, P.P., Aoustin, Y., Gautier, M., Jubien, A., Furet, B.: Accurate force control and co-manipulation control using hybrid external command. In: IFAC World Congress (2017)

3. Khalil, W., Kleinfinger, J., Gautier, M.: Reducing the computational burden of the dynamic models of robots. In: IEEE Int. Conf. on Robotics and Automation, pp. $525-531(1986)$

4. Khatib, O.: A unified approach for motion and force control of robot manipulators: The operational space formulation. IEEE J.on Robotics and Automation 3(1), 4353 (1987). DOI 10.1109/JRA.1987.1087068

5. Khatib, O., Burdick, J.: Motion and force control of robot manipulators. IEEE Int. Conf. on Robotics and Automation 3(5), 1381-1386 (1986). DOI 10.1109/ROBOT. 1986.1087493

6. Morel, G., Bidaud, P.: A reactive external force loop approach to control manipulators in the presence of environmental disturbances. IEEE Int. Conf. on Robotics and Automation 2(April), 1229-1234 (1996)

7. Morel, G., Malis, E., Boudet, S.: Impedance based combination of visual and force control. IEEE Int. Conf. on Robotics and Automation (Cat. No.98CH36146) 2(May), 1743-1748 (1998) 